\title{
Recidiva de macroadenoma hipofisario posterior a tratamiento estándar
}

\author{
Yardany Rafael Mendez-Fandiño* \\ Nelson Javier Perez-Ángel** \\ César Orlando Quintero-Moreno*** \\ Karen Eliana Reyes-Romero*** \\ Claudia Tatiana Salamanca-Velandia** \\ Miguel Ramirez-Rozo****
}

\begin{abstract}
* Médico. Internista. Universidad del Rosario. Servicio de Medicina Interna. Empresa Social del Estado. Hospital Regional de Duitama. Grupo de investigación en Epidemiologia clínica de Colombia (GRECO), Universidad Pedagógica y Tecnológica de Colombia. Tunja. Boyacá. Colombia.

** Estudiante IX nivel de Medicina. Escuela de Medicina. Facultad de Ciencias de la Salud. Universidad Pedagógica y Tecnológica de Colombia. Tunja. Boyacá. Colombia.

*** Estudiante IX nivel de Medicina. Escuela de Medicina. Facultad de Ciencias de la Salud. Universidad Pedagógica y Tecnológica de Colombia. Tunja. Boyacá. Colombia. Grupo de investigación en Epidemiologia clínica de Colombia (GRECO), Universidad Pedagógica y Tecnológica de Colombia. Tunja. Boyacá. Colombia.

****Médico general. Facultad de Medicina. Fundación Universitaria Juan N. Corpas. Bogotá. Cundinamarca. Colombia.

Correspondencia: César Quintero. Escuela de Medicina, Universidad Pedagógica y Tecnológica de Colombia. Avenida Central del Norte 39-115. CP: 150003. Tunja. Boyacá. Correo electrónico: investigacionmedicauptc@gmail.com.
\end{abstract}

\section{RESUMEN}

Introducción: la acromegalia es una patología de larga evolución y compromiso multisistémico, originada por un aumento en la liberación de hormona de crecimiento luego del cierre metafisario de los huesos largos. Su diagnóstico es complejo y suele presentarse entre la quinta y sexta década de la vida. El tratamiento incluye la resección quirúrgica y manejo mediante quimioterapia y radioterapia, así como control endocrino fármacológico. La enfermedad presenta una tasa de recidiva comprendida entre el 2 a 14\%. Presentación de caso: paciente femenina de 67 años de edad con diagnóstico de acromegalia hace 15 años, quien luego de ser sometida a tratamiento estándar, presenta manifestaciones clínicas progresivas y compatibles con una función hipofisaria activa. Conclusiones: la paciente en efecto presentó recidiva de la enfermedad con persistencia del tumor benigno, debido a la interrupción del tratamiento y el insuficiente seguimiento del caso; por tanto, es fundamental el seguimiento estricto en el paciente que recibe manejo quirúrgico y tratamiento farmacológico por parte del equipo de salud. MÉD.UIS. 2017;30(2):89-93.

Palabras clave: Acromegalia. Hormona del Crecimiento. Adenoma Hipofisario Secretor de Hormona del Crecimiento.

\section{Pituitary macroadenoma relapse after standard treatment}

\section{ABSTRACT}

Introduction: acromegaly is a disease of long evolution and multisystemic involvement, caused by an increased release of growth hormone after the metaphyseal closure of long bones. Diagnosis is often difficult and usually occurs between the fifth and sixth decade of life. The treatment is based on surgical resection, chemotherapy and radiotherapy, as on endocrine control by drugs. The disease has a low recurrence rate of from 2 to $14 \%$. Case presentation: a 67 year old female patient with a diagnosis of acromegaly 15 years ago, who, after being subjected to standard treatment, presents progressive and clinical manifestations compatible with an active pituitary function. Conclusions: the patient relapsed effect of the disease with persistency of the benign tumor, due to the discontinuation of treatment and insufficient monitoring of the case; therefore strict monitoring is essential in patients receiving pharmacological therapy and surgical management by the health team. MÉD.UIS. 2017;30(2):89-93.

Keywords: Acromegaly. Growth Hormone. Growth Hormone - Secreting Pituitary Adenoma. 
¿Cómo citar este artículo?: Mendez-Fandiño YR, Perez-Ángel NJ, Quintero-Moreno CO, ReyesRomero KE, Salamanca-Velandia CT. Ramirez-Rozo M. Recidiva de macroadenoma hipofisario posterior a tratamiento estándar. MÉD.UIS. 2017;30(2):89-93.

\section{INTRODUCCIÓN}

La acromegalia es una enfermedad multisistémica crónica, causada por aumento sostenido en la secreción de Hormona de Crecimiento ( $\mathrm{GH}$, por sus siglas en inglés)', la cual se libera normalmente por las células somatotropas de la glándula adenohipófisis ${ }^{2,3}$. Se origina por adenomas hipofisarios alrededor del 95\% de las veces, y por secreción exógena hormonal solo en un $5 \%$. A nivel mundial la prevalencia se estima en 69 casos por millón de habitantes y la incidencia es de tres a cuatro casos por millón de habitantes4; en Colombia, la prevalencia es de 40 a 125 casos por millón de habitantes y la incidencia es similar a la cifra mundial5,6; de cualquier manera, estos datos varían mucho entre distintas regiones del mundo 7 . El proceso diagnóstico de la enfermedad es complejo y prolongado, por lo cual el paciente puede tardar hasta diez años en obtener un diagnóstico definitivo, el cual se presenta más frecuentemente entre la quinta y sexta década de la vida, momento en el cual hasta un $70 \%$ de los tumores se identifican como macroadenomas ${ }^{8,9}$.

El transcurso de la enfermedad implica un efecto deletéreo en el bienestar y la estabilidad emocional del paciente ${ }^{10}$, disminuyendo hasta en diez años la expectativa de vida ${ }^{8}$. La sintomatología principal se basa en crecimiento acral y facial, edema de tejidos blandos, mialgias, atralgias, hiperhidrosis y cefalea. Las manifestaciones clínicas son muy características, por ejemplo, a nivel facial se puede observar un crecimiento pronunciado de la región supraciliar, la nariz y los labios, prognatismo maxilar y macroglosia; en las manos y pies se apreciaría un crecimiento importante de carpo, tarso y falanges ${ }^{1,5}$. De igual forma, puede presentarse acantosis nigricans e hipersecreción sebácea y sudorípara. El crecimiento de los senos paranasales, y la macroglosia ocasionan un deterioro significativo de la función respiratoria, configurando una importante causa de mortalidad ${ }^{10}$.

La enfermedad presenta diversas y frecuentes comorbilidades producto de la afectación sistémica; 25 al $50 \%$ de los pacientes muestran alteración en el metabolismo de los carbohidratos ${ }^{2}$, 18 al $60 \%$ presentan hipertensión arterial crónica ${ }^{5}, 30 \%$ poseen afectación cardiaca ${ }^{6-9}$ (20\% dilatación biventricular y
$10 \%$ insuficiencia cardiaca congestiva), $4 \%$ presenta enfermedad tiroidea ${ }^{11}$ y $20 \%$ presentan osteoporosis u osteoartritis ${ }^{12-13}$. Además, estos pacientes tienen un mayor riesgo de desarrollar panhipopituitarismo. Otros patrones inespecíficos, como neuropatías, edema periférico, amenorrea o disfunción eréctil pueden encontrarse ${ }^{10}$. Por otro lado, el adenoma hipofisario puede causar un efecto compresivo en el quiasma óptico, deteriorando la visión y asociándose a diversas complicaciones ${ }^{8}$, tales como la aparición de meningiomas cerebrales ${ }^{14}$.

El presente artículo describe la recidiva tumoral única en un adulto mayor, posterior a un manejo quirúrgico adecuado y una terapia farmacológica incompleta; donde la resonancia magnética nuclear, evidenció una masa hiperintensa circular delimitada en la silla turca. Todo lo anterior cobra importancia debido a que es fundamental para el médico general y especialista reconocer la existencia de recidiva completa o parcial de la enfermedad, para así vigilar las posibles complicaciones que pueden presentarse, disminuyendo la morbimortalidad y mejorando la calidad de vida del paciente.

\section{Presentación del CAso}

Se presenta el caso de una paciente femenina de 67 años de edad, con antecedentes de hipotiroidismo y panhipopituitarismo secundario a resección de tumor benigno en la silla turca hace 15 años, además con hipertensión arterial, dislipidemia, historial de tres infartos agudos de miocardio, enfermedad pulmonar obstructiva crónica y osteoartritis de cadera. La paciente refirió historial de facies acromegálicas desde hace 35 años, con exacerbación posterior a la resección quirúrgica del tumor de la silla turca y aplicación de radioterapia (Ver Figura 1), y signos sistémicos de afectación ósea (Ver Figura 2). Describió también pérdida del control voluntario de esfínteres, disminución de la agudeza visual, polidipsia y fatiga constante. Al examen físico se evidenció afectación ósea sistémica, principalmente en huesos largos, y macroglosia, asociada a acantosis nigricans en región abdominal y genital, sin otros hallazgos significativos.

Se solicitaron exámenes de laboratorio, detectando TSH de $0,82 \mu \mathrm{U} / \mathrm{ml}(0,35-4,25 \mu \mathrm{U} / \mathrm{ml}$, rango normal 
para la edad de la paciente), prolactina de 27,47 ng/ $\mathrm{ml}(1,9-23 \mathrm{ng} / \mathrm{ml}$, rango normal para la edad de la paciente) y niveles de $\mathrm{GH}$ de $8 \mathrm{ng} / \mathrm{ml}$ ( $5 \mathrm{ng} / \mathrm{ml}$, rango normal para la edad de la paciente). Posteriormente, se realizó examen imagenológico para corroborar la presencia de la masa, en la que se observaron áreas de gliosis frontales mediales, además de una limitada zona de encefalomalacia parasagital frontal derecha y descenso de giros rectos, relacionado con el evento quirúrgico que existe como antecedente; destacó la aparición de masa hiperintensa de bordes lisos y forma redondeada delimitada en proximidad a la silla turca (Ver Figura 3), pero sin que se identificaran signos de reaparición tumoral maligna; como hallazgos complementarios se encontraron cambios de microangiopatía, y de meningioma parafalcino frontal izquierdo y finalmente se sospechó la presencia de aracnoidocele selar.

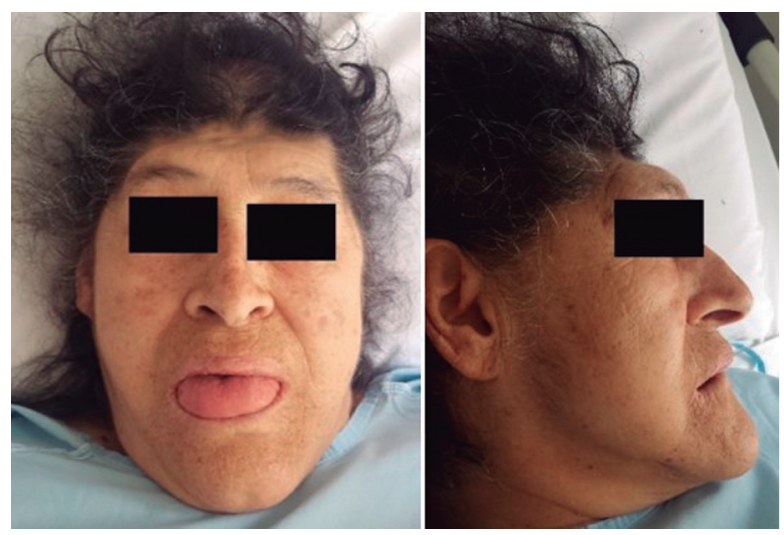

Figura 1: Plano frontal y lateral del rostro, se observa importante crecimiento acral y facial, y macroglosia severa que dificulta la fonación. Por otro lado, se observa hipertrofia de huesos frontales, prognatismo marcado y mala oclusión mandibular. Fuente: autores

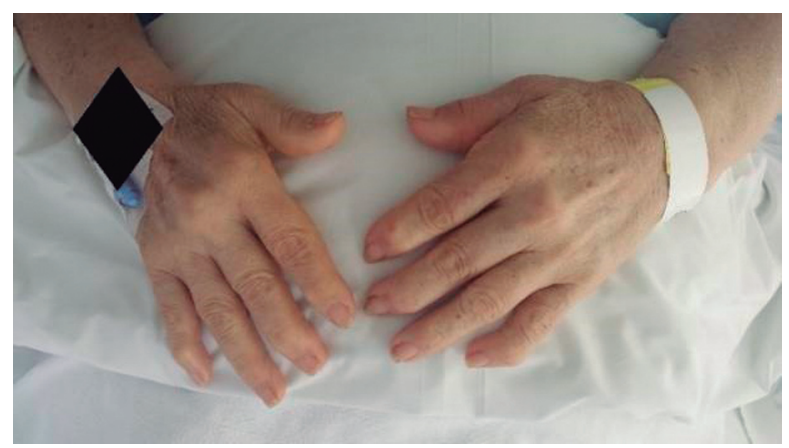

Figura 2: Es posible apreciar aumento del tamaño de falanges de los dedos así como de los huesos del metacarpo. Al igual que de la misma forma. Se aprecia desviación cubital de los dedos Fuente: autores

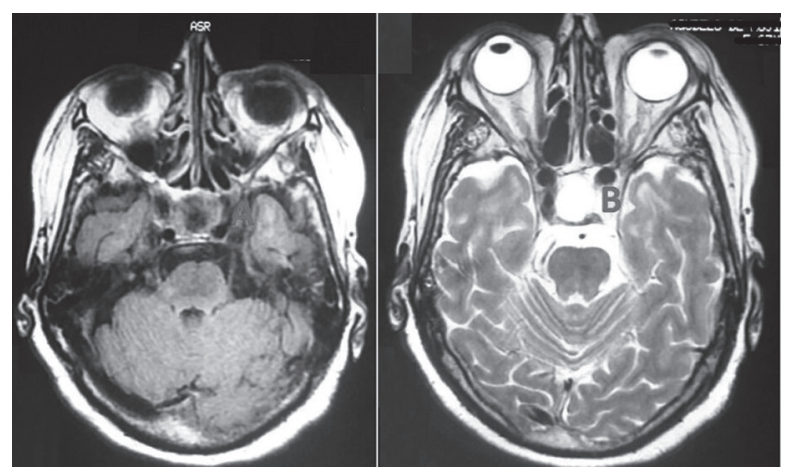

Figura 3: Der. Resonancia Mágnetica Nuclear T2, Se observa reaparición de masa benigna a nivel de la silla turca(B), sin evidencia de malignidad. Izq. Resonancia Mágnetica Nuclear T1 Contrastada: no se observa actividad del contraste con masa tumoral, por lo cual, se dictamina esta como una masa lisa, con bordes definidos, por lo cual tiene un pronóstico benigno.

Fuente: autores

\section{Discusión}

Aunque esta patología puede ser primaria, existen algunos factores que favorecen cambios estructurales y metabólicos a nivel somatotrofico, desencadenando un aumento significativo en la liberación de $\mathrm{GH}$, por ejemplo, la sobreproducción de somatocrinina (hormona liberadora de hormona del crecimiento), o bien un aumento sostenido en la liberación de somatostatina². De igual forma Daly F. y colaboradores han estudiado la relación entre el síndrome de adenomas hipofisarios aislados, con una mutación en el gen de la proteína intercambiadora de hidrocarburos aromáticos ${ }^{15}$, por tanto existe una importante carga genética en el desarrollo de la acromegalia. Es importante resaltar que si la elevación en los niveles de GH se presenta antes del cierre metafisario de los huesos largos, se desarrollara gigantismo hipofisario ${ }^{16}$.

El diagnóstico resulta complejo, ya que en muchas ocasiones el paciente no consulta, atribuyendo la síntomatologia insidiososa a la transición hacia la veje $z^{17}$. La identificación de la enfermedad tiene un alto componente clínico, que se debe complementar con pruebas de laboratorio; el diagnostico se confirma cuando hay ausencia de supresión de GH poscarga oral (75 mg de glucosa)5; o bien cuando se detecta aumento en el valor de somatomedina $C$, también conocida como Factor de Crecimiento Similar a Insulina I (IGF-1), y GH. Cabe mencionar que los valores normales para estas dos hormonas varían de acuerdo a la edad, género y condiciones de salud de base. Los valores normales del IGF-1 van desde 60-160 $\mu \mathrm{g} / \mathrm{L}$ en 
adultos mayores, hasta 190-800 $\mu \mathrm{g} / \mathrm{L}$ en adolescentes; el nivel normal de la GH es de 0 - $5 \mathrm{ng} / \mathrm{ml}(0-5 \mathrm{~g} / \mathrm{L})^{18}$.

La acromegalia es una enfermedad crónica insidiosa causada comúnmente por un adenoma pituitario, su tratamiento es vital por la elevada tasa de mortalidad y sus comorbilidades; este busca:

- Resolver el efecto compresivo del tumor: mediante hipofisectomía, que puede ser transesfenoidal, transcraneal o bien radioterapia7, después de la cual normalmente existe déficit en la producción hormonal².

- Mitigar las manifestaciones clínicas, mediante antagonistas dopaminérgicos como la cavergolina $(0,5-6 \mathrm{mg} / \mathrm{día})^{19}$ o bromocriptina (1,25-2,5 mg/día), análogos de somatostatina como el ocreótido (300 $\mu \mathrm{g} /$ día subcutáneo) o lanreótido (30mg intramuscular cada 28 días); o bien antagonistas del receptor de $\mathrm{GH}$ como el pegvisomant (10-30 mg/día). Existen tratamientos especializados para pacientes embarazadas $^{20}$ y se han desarrollado nuevos medicamentos con diversos mecanismos de acción y mayor seguridad como el pasireotide ${ }^{21}$. Estos medicamentos tienen efecto sintomático exclusivo y son de corta acción, por lo que su uso se debe prolongar hasta que se aborden técnicas quirúrgicas definitivas.

- Normalizar los niveles bioquímicos mediante el manejo de los efectos hormonales periféricos.

- Normalizar la función hipofisaria mediante el control continuo de la función hormonal.

- Igualar la tasa de mortalidad respecto a la población general ${ }^{22}$.

Aunque el abordaje farmacológico es común y el tratamiento de primera línea es la resección vía transesfenoidal, que puede cursar con persistencia de la hipersecreción hormonal en el $43 \%$ de los $\operatorname{casos}^{23}$; se deben considerar nuevas alternativas de manejo, tales como la radiocirugía estereostática, en la cual con el uso de un rayo gamma, que ofrece una sola fracción de radiación a un objetivo pequeño, se delimita a la masa diana limitando la vulnerabilidad de los tejidos blandos adyacentes ${ }^{6,9}$. En un estudio realizado en Polonia para evaluar la eficacia de la neurocirugía, se observó que el 37,6\% de los pacientes se curaban y el $62,4 \%$ requerían tratamiento con análogos de somatostatina ${ }^{24}$.

Después del tratamiento neuroquirúrgico, opción aprobada y aconsejada por el consenso de expertos sobre el tratamiento médico de la acromegalia de la revista Nature Review Endocrinology, se puede implementar la terapia médica con ligandos de receptores de somatostatina como ocreótido y lanreótido y agonistas de dopamina como la cavergolina que han mostrado alta eficacia ${ }^{26}$.Otra opción terapéutica valida es la radioterapia, que permite el control del tumor en un $90 \%$ de los pacientes y la remisión endocrina en un $50 \% 25$, este procedimiento tiene menos efectos secundarios que la radioterapia y está recomendado en pacientes que no responden a la cirugía o terapia farmacológica clásica ${ }^{25}$. En este caso, la paciente presentó recidiva endocrina luego de la radioterapia y la resección quirúrgica, lo cual ocurre en menos del $10 \%$ de los casos en los cuales se presenta macroadenoma hipofisiario ${ }^{25}$.

La paciente es diagnosticada con acromegalia en una fase avanzada y aunque recibe tratamiento quirúrgico y radioterapia, el tratamiento farmacológico es administrado de modo incompleto, razón por la cual se instauran progresivamente signos y síntomas sugestivos de enfermedad recidivante. Además, los niveles de tirotropina y prolactina refuerzan el diagnóstico.

A pesar de la posibilidad baja de recidiva tumoral entre los pacientes afectados por macroadenoma hipofisario secretor de hormona del crecimiento; todo paciente diagnosticado con esta patología, que sea sometido o no a manejo quirúrgico, debe ser objeto de seguimiento clínico continuo y persistente por parte del servicio de endocrinología y neurología, para establecer la evolución final del tumor. De igual forma, si se instaura manejo farmacológico complementario, se debe asegurar su adecuada adherencia por parte del paciente. Con este enfoque, se puede identificar tempranamente, y tratar de manera adecuada, las complicaciones más frecuentes como lo son la compresión quiasmática y secreción hormonal patológica, mejorando así la calidad de vida del paciente.

\section{Conclusiones}

Se presenta el caso de un paciente con recidiva tumoral acompañada de afectación endocrinológica y metabólica continua, después del manejo quirúrgico aprobado. El caso reportado realza la importancia de realizar seguimiento constante y permanente a los pacientes diagnosticados con esta enfermedad, para tratar oportunamente las comorbilidades que puedan presentarse. 


\section{Referencias BibIOGRÁficas}

1. Shlomo M. Medical Progress. Acromegaly. N Engl J Med. 2006;355(24):2558-73.

2. Ruiz R, Duran E, Arellano S, Sanchez V, Moreno O, Mendoza F Acromegalia.Med Int Mex. 2009;25(6):468-80.

3. Shlomo M. Pituitary tumors. Endocrinol Metab Clin N Am. 2015;44(1):1-9.

4. Weslley P, Calsolari M. Screening for acromegaly in adult patients not reporting enlargement of the extremities, but with arterial hypertension associated with another comorbidity of the disease. Arq Bras Endocrinol Metab. 2014;58(8):807-11.

5. Tovar H, Rojas W. Experiencia en acromegalia en el Hospital de San José Bogotá D.C.- Reporte de serie de casos 1990-2007. Acta Med Colomb. 2010;35(2):48-52.

6. Rojas W, Lancheros A, Tapiero M.Descripcion de las alteraciones cardiacas por ecocardiografía de los pacientes con acromegalia. Acta Med Colomb. 2015;40(1):30-5.

7. Abu A, Asi N, Farah WH., Mohammed K, Wang Z, Farah H., et al. Radiotherapy versus radiosurgery in treating patients with acromegaly: A systematic review and meta-analysis. Endocr Prac. 2015;21(8):943-56.

8. Raverot G, Vasiljevic A, Jouanneau E, Trouillas J. A prognostic clinicopathologic classification of pituitary endocrine tumors. Endocrinol Metab Clin N Am. 2015; 44 (1): 11-18.

9. Mayson Sarah E., Snyder Peter J. Silent pituitary adenomas. Endocrinol Metab Clin N Am. 2015;44(1):79-87

10. Crespo I, Valassi E, Santos A, Webb S. Health-Related Quality of life in pituitary diseases. Endocrinol Metab Clin N Am. 2015;44(1):161-70.

11. Paiva H, Balarini G, Lugarinho L, Sieiro A, Aquino S, et al Prevalence of thyroid diseases in patients with acromegaly Experience of a Brazilian center. Arq Bras Endocrinol Metab. 2013;57(9):685-90.

12. Mazziottu G, Chiavistelli, Giustina A. Pituitary diseases and bone. Endocrinol Metab Clin N Am. 2015;44(1):171-80.

13. Bolanowski M, Halupczok J, Jawiarczyk-Przybylowska A.
Pituitary disorders and osteoporosis. J Clin Endocrinol Metab. 2015;(1):1-7.

14. Moncet I. Asociacion simultanea de adenoma hipofisario y meningioma: Reporte de tres casos. Rev. argent. endocrinol. metab. 2015;52(1): 29-34.

15. Daly A, Beckers. Familial isolated pituitary adenomas (FIPA) and mutation in the Aryl hydrocarbon receptor interacting protein (AIP) Gene. Endocrinol Metab Clin N Am. 2015;44(1):19-25.

16. Capatina C, Wass J. 60 Years of neuroendocrinology: Acromegaly. J Endocrinol. 2015;266(2):141-60.

17. Rúa Marín C, Latorre Sierra G, Campuzano Maya G. Diagnóstico de acromegalia. Med Lab. 2011;(11-12):511-31.

18. Junnila RK, Strasburger CJ, Bidlingmaier M. Pitfalls of insulinlike growth factor-i and growth hormone assays. Endocrinol Metab Clin North Am. 2015;44(1):27-34.

19. Auriemma RS, Pivonello R, Ferreri L, Priscitelli P, Colao A. Cabergoline use for pituitary tumors and valvular disorders. Endocrinol Metab Clin North Am. 2015;44(1):89-97.

20. Araujo PB, Vieira Neto L, Gadelha MR. Pituitary tumor management in pregnancy. Endocrinol Metab Clin North Am. 2015;44(1):181-97.

21. Ben-Shlomo A. Pharmacotherapy for acromegaly: future role for pasireotide?. Endocrinol Metab Clin North Am. 2015:44(1):35-41.

22. Katznelson L. Diagnosis and Management of acromegaly in 2014. US Endocrinology. 2014;10(2):120-3.

23. Krzentowska-Korek A, Gołkowski F, Bałdys-Waligórska A, Hubalewska-Dydejczyk A. Efficacy and complications of neurosurgical treatment of acromegaly. Pituitary. 2011;14(2):157-2.

24. Marquez Y, Tuchman A, Zada G. Surgery and Radiosurgery for Acromegaly: A Review of Indications, Operative Techniques, Outcomes, and Complications. International Journal of Endocrinology. 2011;(20):1-7.

25. Del Porto LA, Liubinas SV, Kaye AH. Treatment of persistent and recurrent acromegaly. J Clin Neurosci. 2011;18(2):181-90.

26. Giustina A, Chanson P, Kleinberg D, Bronstein MD, Clemmons DR, Klibanski A, et al. Expert consensus document: A consensus on the medical treatment of acromegaly. Nat Rev Endocrinol. 2014;10(4):243-8. 